\title{
Effect of information asymmetry and em- beddedness on organizational learning
}

\author{
Liefa Liao ${ }^{1}$ Kanliang Wang ${ }^{2}$ \\ ${ }^{1}$ Department of computer science, JiangXi University of Science and Technology, China \\ ${ }^{2}$ Department of management, Xi'an Jiaotong University, China
}

\begin{abstract}
Using simulation research method, this research considers how information asymmetry and embeddedness influence organizational learning performance. The results indicate that, low embeddedness facilitate exploration learning while high embeddedness facilitate exploitation learning in information symmetry network. On the contrary, information asymmetry inhibit exploration learning, further more, the inhibit effect is stronger in embeddedness network than in random network. We discuss the implications and future research direction in the end.
\end{abstract}

Keywords: information asymmetry, embeddedness, organizational learning, network structure

\section{Introduction}

In intensifying competition business environment, learning and adaptation are the major source of competitive advantage [1][2]. In the process of the organizational learning, although the information technology such as data warehouse and knowledge management provide rich source for organizational learning, interpersonal learning among remains the main channel for people to acquire knowledge [3] [4]. In the different types of learning networks, mutual understanding and information symmetry among learners is in different level, and interpersonal relationships embedded in different levels.
It has been shown that information asymmetry and network embeddedness can affect learning process and overall network learning performance. However, the relation between information asymmetry, network embeddedness and organizational learning performance has received little attention. This study advances research on how these two factors affect organizational learning processes. We present an agent-based simulation model that incorporates different information symmetry level and network embeddedness guiding interpersonal learning and evaluate their effects on organizational learning.

This article is organized as follows: Section 2 the hypotheses of this study is given; and then construct and implementation the simulation model; then discussed the simulation results, described the influence of different structures on the learning performance. Finally, we discuss the implications of our findings for management and future research.

\section{Hypothesis}

In the case of information symmetric situation, network learners understand other members' knowledge level. Embedded network is conducive to knowledge and information transfer, enhance trust and improve the effectiveness of a relationship, which is positive to knowledge learning performance. On the other hand, due to the role of friend networks, network members tend to preserve their ex- 
isting knowledge flow channels and reduce the establishment of new learning relationship outside of the acquisition. During this process, learning in this closed network serves to eliminate differences between the individuals. Consequently, the knowledge of individuals converges over time. As individuals in the network become more knowledgeable, their knowledge also becomes more homogeneous. Equilibrium is reached at which all network member share the same knowledge with respect to closed learning network and effective knowledge transfer within it.

In contrast, in low embedding learning networks, learner explores learning objects in random mode, this randomness reduces the probability of successful learning, and it reduces efficiency of the internal network knowledge transfer. Furthermore, the relationship embeddedness level between learning objects is lower than high embedded networks which will low efficiency of knowledge learning. Thus, the friend relationship can transfer knowledge quicker than the random network. On the other hand, random selected learning objects can keep knowledge heterogeneity for longer time, and the change of whole network knowledge is slower than high embedded network, which make learning in network in an exploration approach. Based on this, we assume:

Hypothesis 1: In the symmetric information networks, high-embedded network is more beneficial in exploitation learning than random network, while random networks conducive to exploration learning.

In information asymmetric network, the network members are difficult to understand the specific knowledge of others, which make heuristic information such as accessibility and social influence become the main information for learner to select learning object. While this heuristic in- formation may have some inconsistencies with true knowledge of network member, the selected learning objects with high social influence may not have higher knowledge level than learner. Then the learning relationship may not have an effective knowledge transfer, in this situation, heuristic information reduces learning efficiency.

On the other hand, in the case of learning failure, the learners will gradually forget the existing useless learning relationship and try to build new learning relation $[5,6]$, during this process, learners tend to maintain social relations with high influence personnel, and interrupt their learning relationship with inferior social influence and low accessibility node. In addition, during the process of establishing new learning relationships, learners are still to find a new learning object according to heuristic information in learning network under information asymmetry situation.

In this process, there may be a lot of learning relationship which exist stable in these learning networks, not realize knowledge transfer and inhibit the formation of the new network connection. In the same time, as the learning relationships establishment rely on heuristic information, network members which have high knowledge level and low social influence can hardly be recognized by others, which reducing the opportunity for sharing knowledge, and become a high social influence person in learning network.

The interactive affect of these two factors make the learning network showed a low learning speed, and preserving a high level of knowledge heterogeneity. Based on this, we assume:

Hypothesis 2: the asymmetry of information negative affects short-and longterm learning performance.

In addition, in the knowledge information asymmetry learning networks, high- 
embedded network enables learners finding high social influence learning objects, building high social impact learning network, reducing the influence from other low social influence network members. As above discussed, high social impact does not necessarily mean high knowledge level, which make embedded network increase the risk of learning failure. In the same time, in random learning networks, the speed which learners search for high-impact learners is lower than that friend's network, which increases the possibility of learning from other low social impact and high knowledge network members, and improves the overall network learning performance. Based on this, we assume:

Hypothesis 3: the negative affect of information asymmetry on knowledge learning performance is higher in embeddedness network than in random network.

\section{METHODOLOGY}

We conduct this research using simulation. Simulation is a particularly effective method for research where the basic outline of the theory is understood, but its underlying theoretical logic is limited. While research phenomenon is non-linear and empirical data are challenging to obtain[7][8].

For organizational learning, March [9] built a simulation model to explore the learning speed, mobility and the external environment changes on the level of organizational knowledge, followed by Miller and other extensions of the simulation model (such as [10-12]). Thus, the paper builds interpersonal learning simulation model in the network base on this simulation model. We regard an organization as a complex adaptive system, where individuals interact with other individuals. In particular, we view individuals as carriers of ideas and knowledge, and organizational learning as a property that emerges from interactions among individuals in the organization.

The simulated organization's goal is to maintain the best possible representation of an exogenous environment. Our model has six main entities.

(1). External Reality. We describe reality as having $\mathrm{m}$ dimensions, each of which has a value of 1 or -1 . The probability that any one dimension will have a value of 1 (or -1 ) is 0.5 .

(2). Individual knowledge. There are $n$ individuals in an organization. Each individual holds beliefs about the environment in an m-element vector at each time step. A value of 0 reflects the absence of a belief about a particular dimension of the organization environment.

In the simulation, individual knowledge is defined as the extent to which a belief structure matches the exogenous real world. An individual's knowledge is calculated as:

$$
I K_{i}=\sum_{j=1}^{m}\left(b_{j}^{i} * e_{j}\right)
$$

The average level of individual knowledge is given by

$$
A O K_{i}=\frac{1}{n} \sum_{i=1}^{n} \sum_{j=1}^{m}\left(b_{j}^{i} * e_{j}\right)
$$

This measure is bounded at +1 and -1 . The upper bound, 1, indicates complete correspondence between all individuals' belief structures and the environment.

(3). Knowledge update method. During the simulation, the knowledge update method is calculated as

$$
\text { Bi.t=Bi.t-1*(1-p)+SuperKN*p }
$$

Where: Bi.t-1 is i individual's knowledge vector in t-1cycle; Bi.t is individual's knowledge vector in $\mathrm{t}$ cycle, SuperKN is high knowledge vector; $p$ is learning speed.

(4). Learning decision-making process 
In the knowledge learning process, learners make decision of whether to establish new learning relationships based on last cycle learning situation.

If learner gets new knowledge from learning object successful in last cycle, he will maintain existing learning networks, and does not change the network structure. On the contrary, if learner do not learn what he want in the last cycle, he will expand the scope of its learning network, and try to establish a new relationship, in the same time, in order to avoid overload of maintain learning relationship, the learner will delete an existing useless learning connections.
When establishing new learning relationship, learner will use different select strategy in different situation according to different information symmetry and network embeddedness. Firstly, learner in a random network will selects learning candidate randomly, and will select the most closely connection person from the existing connection in the friend connect network. Secondly, in information asymmetry situation, the learner will select the learning candidate base on the accessibility [13], and select learning candidate by their true knowledge level in symmetry situation. Figure 1 shows network changing process discussed above.

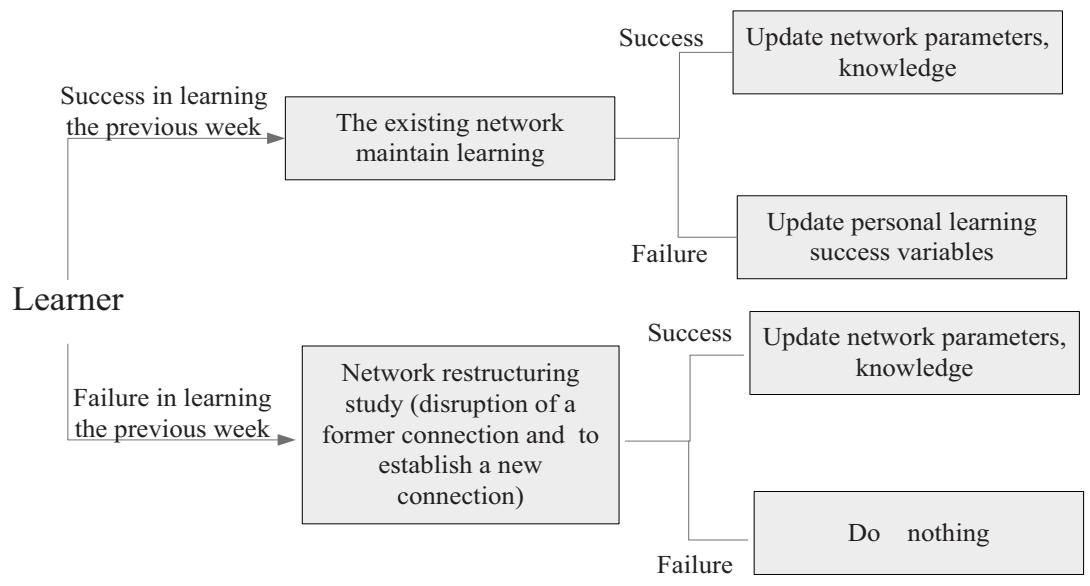

Fig. 1: The learning relationship update decision process.

(5). Relationship dynamics

Relationship Enhancement: during learning process, the interaction between network members will affect relationship strength. In successful learning situation, the relationship between the nodes can become stronger in both sides, and enhance satisfaction and improve mutual learning possibilities between them in the future.

Relation decay: In the individual learning process, each person maintains a certain number of active connections, and he will reduce or forgotten useless learning relations, we express relationship dynamics in following way:

$$
L S_{i j}^{t}= \begin{cases}L S_{i j}^{t-1}+1 & \text { if learn }{ }_{\mathrm{ij}}^{\mathrm{t}-1}=\text { ture } \\ L S_{i j}^{t-1} & \text { if }\left(\mathrm{KL}_{\mathrm{j}}^{\mathrm{t}-1}>=\mathrm{KL}_{\mathrm{i}}^{\mathrm{t}-1} \| \mathrm{SL}_{\mathrm{j}}^{\mathrm{t}-1}>=S \mathrm{~L}_{\mathrm{i}}^{\mathrm{t}-1}\right) \& \&\left(\operatorname{learn}_{\mathrm{ij}}^{\mathrm{t}-1}=\text { false }\right) \\ L S_{i j}^{t-1}-1 & \text { if }\left(\mathrm{KL}_{\mathrm{j}}^{\mathrm{t}-1}<\mathrm{KL}_{\mathrm{i}}^{\mathrm{t}-1} \| \mathrm{SL}_{\mathrm{j}}^{\mathrm{t}-1}<S \mathrm{~L}_{\mathrm{i}}^{\mathrm{t}-1}\right) \& \&\left(\operatorname{learn}_{\mathrm{ij}}^{\mathrm{t}-1}=\text { false }\right)\end{cases}
$$


LS: connection strength in time t. KL: knowledge level

SL: social impact of node. Learn t-1 : Learnt-1: the learning situation in time $\mathrm{t}-1$.

(6). Parameter setting and simulation process

We constructed our simulation model for each set of parameter specifications (table 1). In the initialization stage, set- ting the relevant simulation parameters, and individuals were randomly assigned to the $\mathrm{n}$ m-dimensional knowledge vector. For each run of the model, we calculated the average knowledge level of all individuals. On completing specification analysis, we focused the discussion on the simulation result related to organizational learning performance.

\begin{tabular}{|c|c|c|c|}
\hline Parameter & Meaning & Parameter values & sensitivity analysis \\
\hline $\mathrm{N}$ & The number of individual team & 50 & $20,30,80,150$ \\
\hline $\mathrm{M}$ & Dimension of knowledge & 200 & $120,150,250,300$ \\
\hline $\mathrm{S}$ & $\begin{array}{c}\text { The number of learning dimension of } \\
\text { knowledge }\end{array}$ & 20 & $10,30,50,70$ \\
\hline $\mathrm{P}$ & $\begin{array}{c}\text { Interpersonal knowledge and learning } \\
\text { speed }\end{array}$ & 0.5 & $0.1,0.3,0.7,0.9$ \\
\hline $\mathrm{K}$ & $\begin{array}{c}\text { Individual maximum number of con- } \\
\text { nections (learning objects) }\end{array}$ & 5 & $3,8,10,15$ \\
\hline
\end{tabular}

Table 1: Parameter value in simulation process.

\section{Results}

Figure 2 shows that different levels of information symmetry and embeddedness may affect the level of organizational learning processes and performance significantly.

In the initial stage of simulation, the speed of knowledge improving and speed of knowledge variance reduction are most fast in information symmetry and embeddedness network, and the learning processing is in exploitation learning style. In contrast, the knowledge enhances speed and knowledge variance reduction speed is slower in information symmetry and random search networks. We also can seen from figure 2 that in information symmetric situation, the variance level of network knowledge will eventually reduced to very low level within the network members and reach high level of homogeneity. This shows that in information symmetric case, embeddedness network more positive affect exploitation learning than random search network.

On the other hand, in information asymmetric case, the knowledge improving speed slower than information symmetric situation significantly, the network will achieve stable and low level network knowledge. By analyzing the differences of these two situation, we can see that knowledge variance maintain at a high level within the information asymmetric network, so that members of the network is at a learning standstill states, the network did not achieve adequate learning, information asymmetry hindered effective communication between the different levels of knowledge network member.

In addition, the simulation results also show that under information asymmetric situation, different levels of network embeddedness also affect knowledge learning performance, comparing knowledge level in random networks and embeddedness network, we can see knowledge level is higher in information asymmetry network, this means that the affect of ad- 
verse selection is lower in learning network. At this point, the existing relations

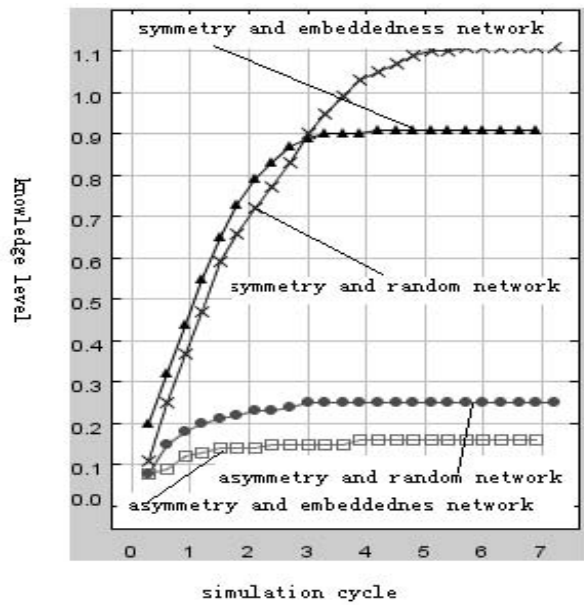

in network are in the role of disservice.

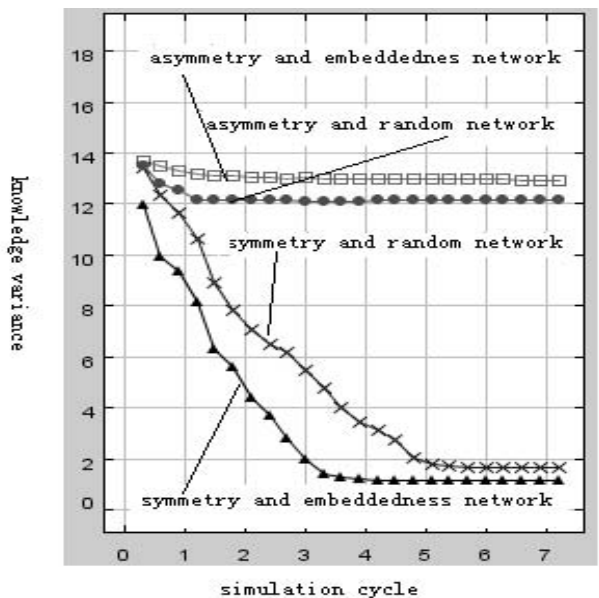

Fig. 2: Information asymmetry, embeddedness and knowledge level in network.

\section{Discussion}

This study constructed a dynamic simulation model to explore the effect of information asymmetric and network embeddedness on the organizational learning performance. The results confirmed that information asymmetry and network embeddedness affect organizational learning performance, low embeddedness facilitate exploration learning while high embeddedness facilitate exploitation learning in information symmetry network. On the contrary, information asymmetry inhibit exploration learning, further more, the inhibit effect is stronger in embeddedness network than in random network. Based on this study, the conclusion of this article can be verified by longitudinal empirical study in the future.

\section{References}

[1] Tsai, W., Knowledge Transfer In Intraorganizational Networks: Effects Of Network Position And Absorptive
Capacity On Business Unit Innovation And Performance $[\mathrm{J}]$. Academy Of Management Journal, 2001. 44(5): P. 996-1004.

[2] Schulz, M., The Uncertain Relevance Of Newness: Organizational Learning And Knowledge Flows[J]. Academy Of Management Journal, 2001. 44(4): P. 661-681.

[3] Cross, R., S.P. Borgatti, And A. Parker, Beyond Answers: Dimensions Of The Advice Network, In Social Networks. 2001. P. 215-235.

[4] Nebus, J., Building Collegial Information Networks: A Theory Of Advice Network Generation[J]. Academy Of Management Review, 2006. 31(3): P. 615-637.

[5] Burt, R.S., Attachment, Decay, And Social Network[J]. Journal Of Organizational Behavior, 2001. 22(6): P. 619-643.

[6] Burt, R.S., Decay Functions, In Social Networks. 2000. P. 1-28.

[7] Davis, J.P., K.M. Eisenhardt, And C.B. Bingham, Developing Theory Through Simulation Methods[J]. 
Academy Of Management Review, 2007. 32(2): P. 480-499.

[8] Harrison, J.R., Et Al., Simulation Modeling In Organizational And Management Research[J]. Academy Of Management Review, 2007. 32(4): P. 1229-1245.

[9] March, J.G., Exploration And Exploitation In Organizational Learning $[\mathrm{J}]$. Organization Science, 1991. 2(1): P. 71-87.

[10] Davis, J.P., Eisenhardt, K. M., \& Bingham, C. B, Optimal Structure, Market Dynamism, And The Strategy Of Simple Rules [J]. Administrative Science Quarterly, 2009.
[11] Rahmandad, H., Effect Of Delays On Complexity of Organizational Learning[J]. Management Science, 2008. 54(7): P. 1297-1312.

[12] Miller, K.D., Z. Meng, And R.J. Calantone, Adding Interpersonal Learning And Tacit Knowledge To March's Exploration-Exploitation Model[J]. Academy Of Management Journal, 2006. 49(4): P. 709-722.

[13] Atuahene-Gima, K., Resolving the capability-rigidity paradox in new product innovation, in Journal of Marketing. 2005. p. 61-83. 